\title{
On the ion distributions at the separatrices during symmetric magnetic reconnection
}

\author{
HongTao Huang ${ }^{1}$, YiQun $\mathrm{Yu}^{1,2 *}$, JinBin Cao ${ }^{1,2}$, Lei Dai ${ }^{3}$, and RongSheng Wang ${ }^{4}$ \\ ${ }^{1}$ School of Space and Environment, Beihang University, Beijing 100191, China; \\ ${ }^{2}$ Key Laboratory of Space Environment Monitoring and Information Processing, Ministry of Industry and Information Technology, Beijing 100191, China; \\ ${ }^{3}$ State Key Laboratory of Space Weather, National Space Science Center, Chinese Academy of Sciences, Beijing 100190, China; \\ ${ }^{4}$ Key Laboratory of Geospace Environment, Department of Geophysics and Planetary Science, University of Science and Technology of China, Hefei 230026, \\ China
}

\section{Key Points:}

- Ion distribution on the separatrices exhibits two counter-streaming cores, with two discrete populations in the parallel direction.

- The four populations are formed by ions transported from different regions near the reconnection site.

- Kinetic energy of ions converted from the electromagnetic energy is contributed mainly by the Hall electric field.

Citation: Huang, H. T., Yu, Y. Q., Cao, J. B., Dai, L. and Wang, R. S. (2021). On the ion distributions at the separatrices during symmetric magnetic reconnection. Earth Planet. Phys., 5(2), 205-217. http://doi.org/10.26464/epp2021019

\begin{abstract}
A particle-in-cell simulation of symmetric reconnection with zero guide field is carried out to understand the dynamics of ions along the separatrices. Through the investigation of ion velocity distributions at different moments and locations along the separatrices, a typical distribution is found: two counter-streaming populations in the perpendicular direction, with another two populations accelerated into distinct energy levels in the parallel direction. Backward tracing of ions reveals that the counter-streaming cores are mostly composed of ions initially located at the same side of the separatrix, while the other two accelerated populations in the parallel direction are composed of ions crossing through the neutral sheet. Through analysis of energy conversion of these populations, it is found that the ion energization along the separatrix is attributable primarily to the Hall electric field, while that in the region between the two separatrices is caused primarily by the induced reconnection electric field. For the counter-streaming population, the low-energy ions that cross the separatrix twice are affected by both Hall and reconnection electric fields, while the high-energy ions that directly enter the separatrix from the unperturbed plasma are energized mainly by the Hall electric field. For the two energized populations in the parallel direction, the ions with lower-energy are accelerated mainly by the in-plane electric field and the Hall electric field on the opposite side of the separatrix, whereas the ions with higher-energy not only experience the same energization process but also are constantly accelerated by the reconnection electric field.
\end{abstract}

Keywords: magnetic reconnection; particle-in-cell simulation; energetic particles

\section{Introduction}

Magnetic reconnection is a basic plasma process in the space environment that results in plasma heating and acceleration with an explosive release of magnetic energy (Dungey, 1961; Yamada et al., 2010). The present understanding of magnetic reconnection is based primarily on a two-dimensional picture, in which the physical process of reconnection can be divided into different regions. The central site of reconnection is commonly named the " $X$ line", at which oppositely directed magnetic field lines are reconnected. The vicinity of the reconnection $X$ line is found to be capable of producing energetic particles. For instance, Hoshino et al. (2001) found that electrons are accelerated around the $X$ line region due

Correspondence to: Y. Q. Yu, yiqunyu17@gmail.com

Received 06 SEP 2020; Accepted 04 JAN 2021.

Accepted article online 04 FEB 2021.

C 2021 by Earth and Planetary Physics. to the meandering motion in zero guide field reconnection. For guide field reconnection, Fu XR et al. (2006) proposed that strong guide field can keep the electrons longer in the $X$ line region, and thus the acceleration is enhanced. The $X$ line is described as being surrounded by an electron diffusion region and an ion diffusion region. A diffusion region is one in which inflowing particles decouple from the magnetic field, breaking the frozen-in condition. The electron diffusion region is embedded within the ion diffusion region. Previous studies have proposed many prominent features inside the diffusion regions (e.g., Shay et al., 2016). The electron diffusion region can be divided into two regions (Karimabadi et al., 2007): the inner region is identified by a strong out-of-plane electron current while the outer region features highly collimated electron jets that are gradually decelerated and thermalized. Within the electron diffusion region, the energy dissipation is enhanced due to non-ideal energy conversion (Zenitani et al., 2011). The crescent-shaped electron distribution residing inside the elec- 
tron diffusion region has recently attracted much attention, and is suggested to be attributable to the meandering motion of electrons in the magnetic field reversal (Hesse et al., 2014; Chen LJ et al., 2016; Lapenta et al., 2017).

Whereas electrons flow during reconnection mainly from the upstream region through the diffusion region into the downstream region, ions, too, flow across the ion diffusion region into the downstream region, but most ions enter the downstream region through the separatrices. Separatrices are layers that develop from the $X$ line, separating the reconnected region from the unreconnected region. Recent studies have found that the separatrices are highly structured; they can contain sub-regions such as the sub-ion region, the sub-electron region (Zhou $M$ et al., 2012), and the flux ropes (Daughton et al., 2011; Huang SY et al., 2016a), and are important sites for waves and instabilities to be excited. Dai L (2009), from the theoretical point of view, proposed that the Hall fields on the separatrices are components of kinetic Alfvén wave eigenmodes. Particle-in-cell simulations revealed that these Hall fields can transmit along the magnetic field line as kinetic Alfvén waves (Liang J et al., 2016; Huang HT et al., 2018). Fujimoto (2014) reproduced the electromagnetic waves near the separatrices in particle-in-cell simulation and found that the waves are generated mainly by electron beams. Goldman et al. (2014) proposed that the emission of quasi-parallel whistler waves is created by electron phase-space holes on the separatrices through particle-in-cell simulations, which has been confirmed by Huang SY et al. (2016b) through a case study by using the Cluster data. Moreover, based on a statistical study by Huang SY et al. (2017), the separatrix region is found to be more favorable for the excitation of whistler waves than the vicinity of the $X$ line. Furthermore, separatrices are found to have the potential to effectively accelerate particles. Drake et al. (2005) suggested that electrons can be accelerated to relativistic energy due to the parallel electric field on the separatrices. Wang RS et al. (2013), through Cluster measurements, found that electrons are accelerated up to $100 \mathrm{keV}$ on the separatrices.

While electron behavior in the reconnection have been widely studied in previous studies, including the acceleration of electrons in different regions - such as the dipolarization fronts (Zhou M et al., 2009; Fu HS et al., 2012; Huang C et al., 2015) and the magnetic islands (Huang SY et al., 2012; Wang HY et al., 2016; Wang HY et al., 2017), and the electron crescent-shaped distribution in electron diffusion region (Hesse et al., 2014; Burch et al., 2016; Chen LJ et al., 2016; Shay et al., 2016; Lapenta et al., 2017), the behavior of ions, which has been found to be significantly different from that of the electrons, due to their larger gyroradius, has been much less investigated. Recent studies suggest that ions could gain more energy in reconnection than electrons, indicating that ions may play a vital role in magnetic reconnection. Yamada et al. (2014), through a laboratory reconnection experiment, quantitatively compared the energy conversion between ions and electrons in a well-defined reconnection layer, and found that when half of the magnetic energy is converted to particle energy, the ions gain twice as much energy as electrons. Through fully kinetic simulations, Li XC et al. (2015) found that among the energy converted from magnetic energy to kinetic energy, ions gain
1.6 times as much energy as do electrons. Wang $S$ et al. (2018) investigated the energy conversion and partition by means of particle-in-cell simulations and found that the farther away from the $X$ line, the more energy ions gain compared to energy gained by electrons. They also reported a magnetopause reconnection event observed by the Magnetosphere Multiscale (MMS) mission that confirmed the simulation results. Ion dynamics have also been reported in previous studies. For instance, a case study by Zhou XZ et al. (2010) of Time History of Events and Macroscale Interactions during Substorms (THEMIS) observations reported that ions are reflected and accelerated by the dipolarization fronts. They also performed test-particle simulations to reproduce the observed ion distributions. Using test-particle simulations and theoretical models, Zhou XZ et al. (2009) and Zhou XZ et al. (2016) analyzed the ion distributions observed by THEMIS in a reconnection event and found that mushroom-shaped distributions are developed due to the effect of the finite ion gyroradius. Wygant et al. (2005) proposed that the Hall electric field on the separatrices produces a potential well structure and can accelerate ions ballistically. Aunai et al. (2011), using 2-D hybrid simulation, found that ions can bounce between the potential well structure associated with the Hall electric field on the separatrices, and the potential electric energy is transferred to kinetic energy. Wang $S$ et al. (2016), using 2-D particle-in-cell simulation and MMS observations, investigated ion velocity distributions in the two-scale structure of the ion diffusion region in asymmetric magnetic reconnection and found that ions exhibit counter-streaming beams in the inner scale and exhibit crescent shapes in the outer scale.

To further our understanding of the ion dynamics in the reconnection region, unraveling how the ion distributions are developed on the separatrices is significant given its critical role in the energy conversion. In this study, we aim to advance understanding of the ion distributions on the separatrices by particularly investigating their evolution as the reconnection progresses, as well as their source regions and energization along the paths. Such a study may shed light on the fate of the particles near the reconnection site and deepen our knowledge of magnetic reconnection.

\section{Simulation Setup}

The simulation applies the fully electromagnetic implicit particlein-cell code iPIC3D (Markidis et al., 2010). The initial condition is a Harris current sheet with no guide field, which has proved versatile in previous studies (Huang HT et al., 2018). The background magnetic field is given by:

$$
B_{x}=B_{0} \tanh \left(\frac{y}{a}\right)
$$

and the density is given by

$$
n=n_{0} \cosh ^{-2}\left(\frac{y}{a}\right)+n_{\mathrm{b}}
$$

where $a=0.5 d_{\mathrm{i}}\left(d_{\mathrm{i}}\right.$ is the ion inertial length) is the half thickness of the current sheet, $B_{0}=30 \mathrm{nT}$ is the asymptotic magnetic field applicable to the magnetotail, and $n_{0}=0.05 \mathrm{~cm}^{-3}$ is the density at the center of the initial current sheet. Particles consisting of the current sheet are initialized as a drifting Maxwellian velocity distribution. The background particles are introduced with density $n_{\mathrm{b}}$ 
set to be $0.1 n_{0}$, and are initialized with a Maxwellian velocity distribution. Due to the limitation of computational resources, the artificial mass ratio $m_{\mathrm{i}} / m_{\mathrm{e}}=100$ is employed, which is commonly used in previous studies regarding the dynamics of ions in magnetic reconnection (Drake et al., 2009). The simulation is conducted in the 2-D $X Y$ plane $(\partial / \partial Z=0)$. The simulation box size is $L_{x} \times L_{y}=120 d_{\mathrm{i}} \times 40 d_{\mathrm{i}}$ with $3072 \times 1024$ grid points in the computational domain. There are 1024 electron-ion pairs of particles initially launched in each cell. The time step is $\omega_{\mathrm{pi}} t=0.125\left(\omega_{\mathrm{pi}}\right.$ is the ion plasma frequency). The boundary conditions are periodic in $X$, while on the $Y$ boundaries particles are reflected and field conditions are conducting. Magnetic reconnection is initiated with a small perturbation located in the center of the box (Huang HT et al., 2018).

\section{Simulation Results}

When the reconnection starts to evolve, oppositely directed magnetic field lines merge and the separatrices develop from the re- connection site. As reconnection evolves, the topology of magnetic field lines changes; the separatrices expand during this process. The remarkable signature of the separatrices is the concentration of strong Hall electric field and Hall magnetic field. Figure 1 shows the temporal evolution of the three electric field components around the separatrices. Both the in-plane electric field $E_{x}$ and the Hall electric field $E_{y}$ are mainly concentrated on the separatrices but the latter is dominant. Moreover, the Hall electric field $E_{y}$ transmits downstream with the evolution of the reconnection, directing towards the current layer. This scenario is consistent with previous studies (Dai L, 2009, 2018; Dai L et al., 2017; Huang HT et al., 2018), where the Hall electric field $E_{y}$ in magnetic reconnection is identified as the kinetic Alfvén wave. Unlike $E_{x}$ and $E_{y}$, the strongest induced reconnection electric field $E_{z}$ dominates the outflow region enclosed by the separatrices, implying that these electric fields can accelerate particles at different regions. We investigate ion distributions along the separatrices at four locations, each with a size of $0.5 d_{\mathrm{i}} \times 0.5 d_{\mathrm{i}}$. The four regions are: the
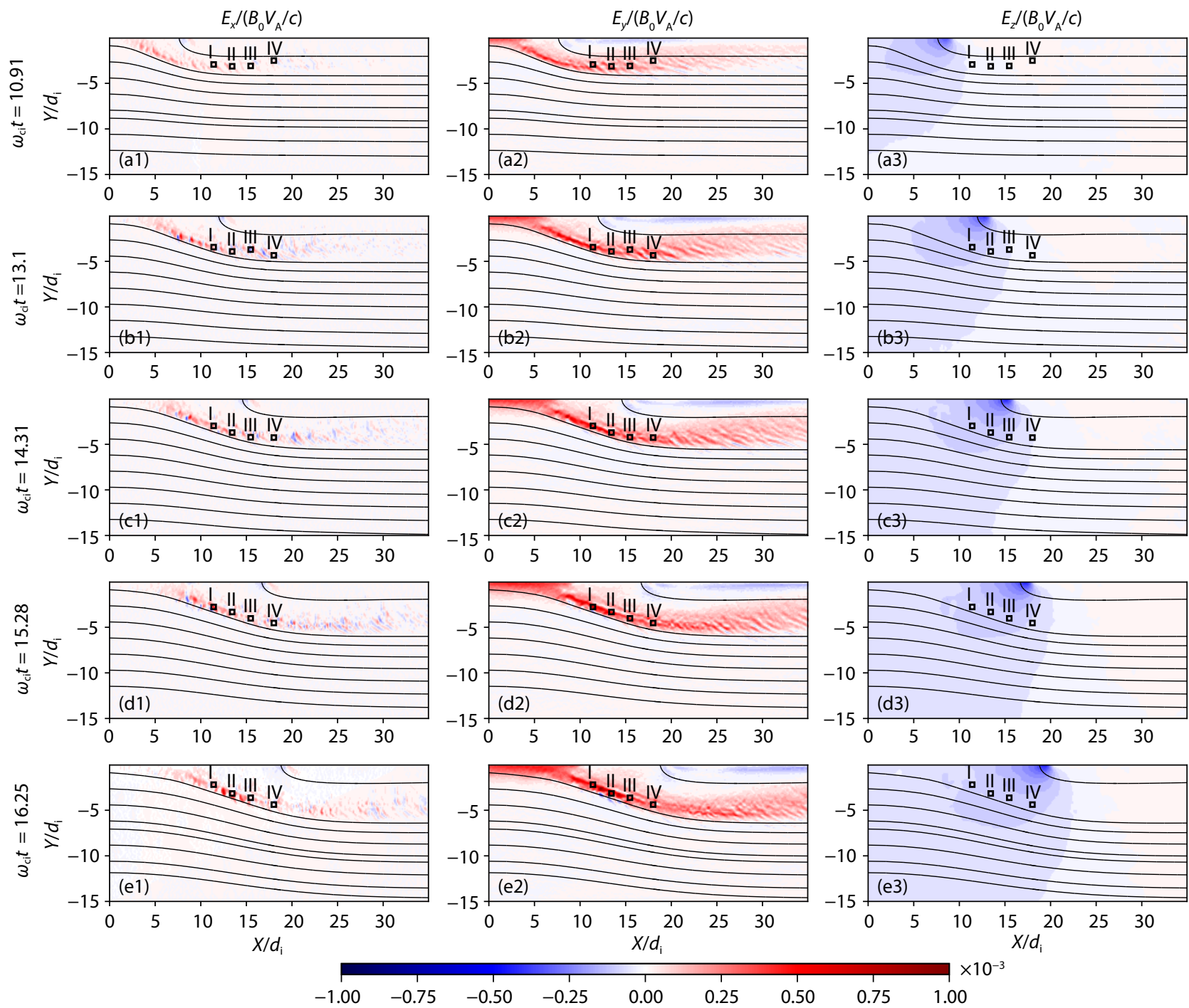

Figure 1. The three electric field components around the separatrix, normalized to $B_{0} V_{\mathrm{A} 0} / c$. Five moments are chosen corresponding to different phases along the reconnection process. A quarter of the typical reconnection box is shown. The black lines indicate the magnetic field lines. Four boxes for detailed analysis of the ion velocity distributions are marked with black rectangles. 
source region close to the $X$ line $\left(X: 11.1-11.6 d_{\mathrm{i}}\right)$, marked as Region l; the region between the source region and the downstream of the separatrix, denoted by Region II $\left(X: 13.2-13.7 d_{\mathrm{i}}\right)$ and Region III $\left(X: 15.2-15.7 d_{\mathrm{i}}\right)$; the downstream of the separatrix $\left(X: 17.7-18.2 d_{\mathrm{i}}\right)$, denoted as Region IV. Note that the location of separatrices changes in time; that is, it expands in the $Y$ direction after the reconnection is triggered. As shown in Figure 1, our selection of $Y$ position is adaptively changed during the expansion of the separatrices while examining the ion distributions. This means that the subsequent analysis of ion dynamics within these four regions depends on the specific location at different moments.

\subsection{Ion Velocity Distributions at Different Locations and Moments}

We first look at the ion bulk motion around the separatrices. As shown in Figure 2, the positive ion bulk velocity $v_{x}$ in the outflow region denotes the reconnection outflow jet while the positive $v_{y}$ indicates that a large quantity of ions flows across the sep- aratrices into the downstream. We investigate the ion velocity distribution in a 3-D velocity space: (1) the parallel velocity, parallel to the background magnetic field; (2) the perpendicular component obtained by the cross product of the background magnetic field and the ion bulk velocity, denoted as the $V_{\perp 1}$ component; and (3) the other perpendicular component forming a cartesian coordinate system, denoted as the $V_{\perp 2}$ component. It should be noted that the magnetic field line curvature radius is much larger than the ion gyroradius around the separatrix, so that this coordinate system is applicable.

Figure 3(a-d) show the ion velocity distributions in Regions I-IV, respectively, at $\omega_{\text {ci }} t=15.28$ when reconnection is well developed and the reconnection rate is steady. The first column in Figure 3 shows the history of Hall electric field intensity at these four regions, which serves as an indicator of the reconnection process there. The red dashed line indicates the time at $\omega_{\mathrm{ci}} t=15.28$. Note that as the separatrix expands, these four locations that are selected at $\omega_{\mathrm{ci}} t=15.28$ do not always lie on the separatrix, as can be seen in Figure 1. Before $\omega_{\mathrm{ci}} t=15.28$, these locations are off the
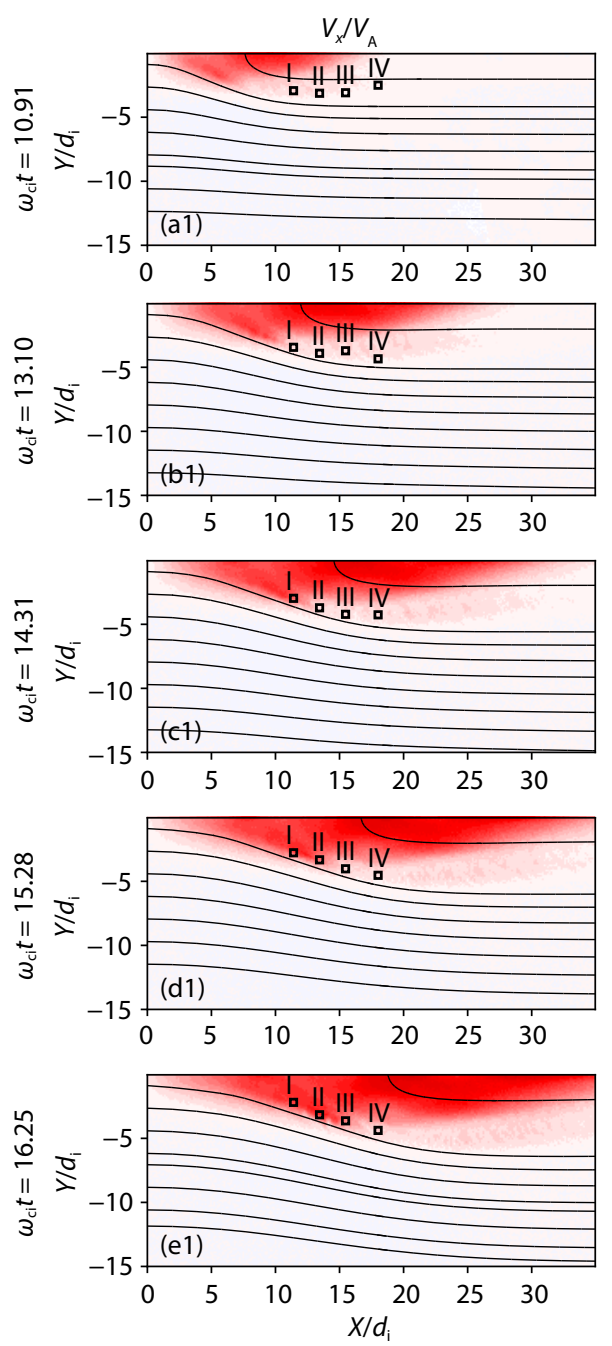
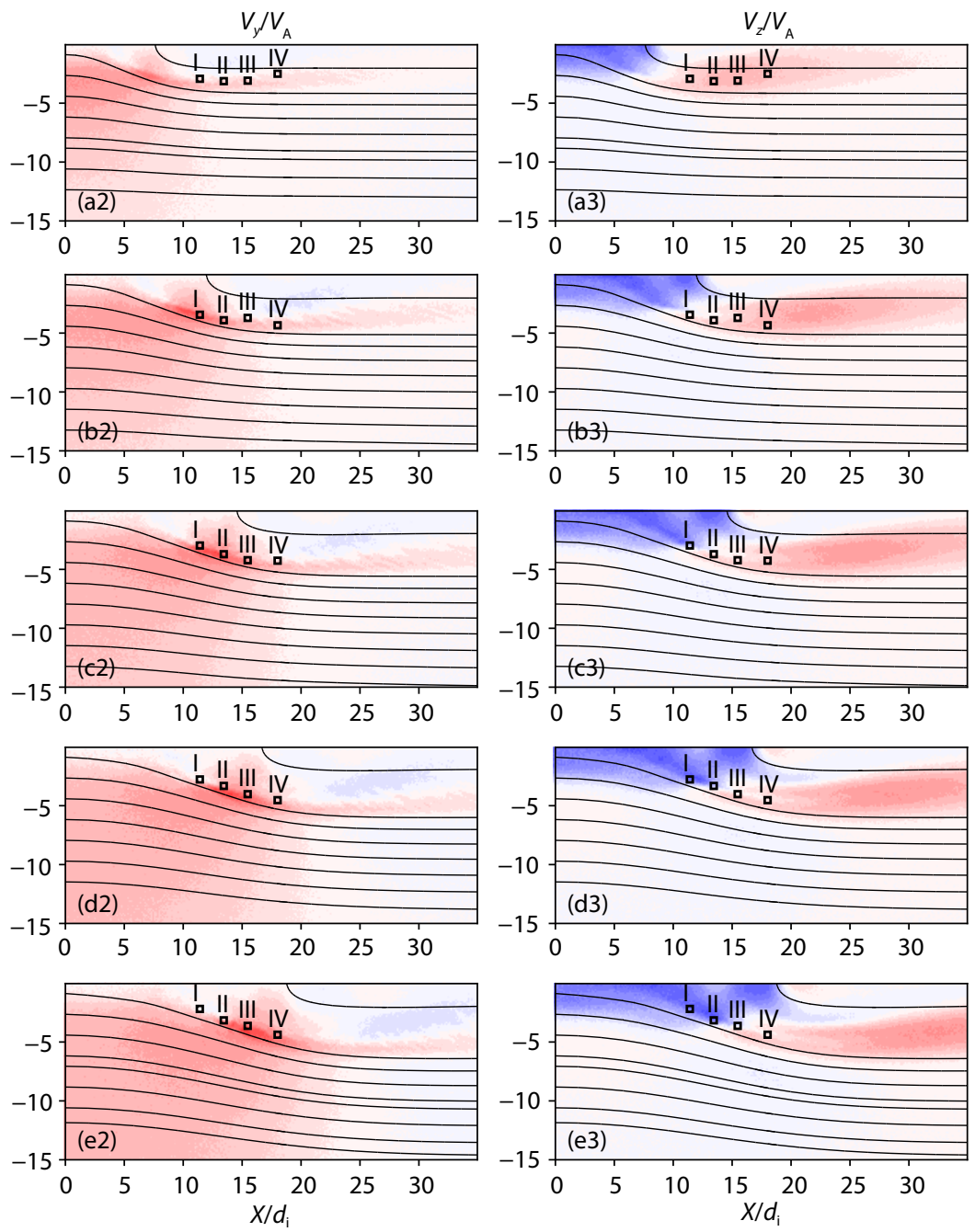

$-2$

$-1$

0

2

Figure 2. The ion bulk velocity around the separatrix, normalized to $V_{\mathrm{A} 0}$. Figure 2 is in the same format as Figure 1. 

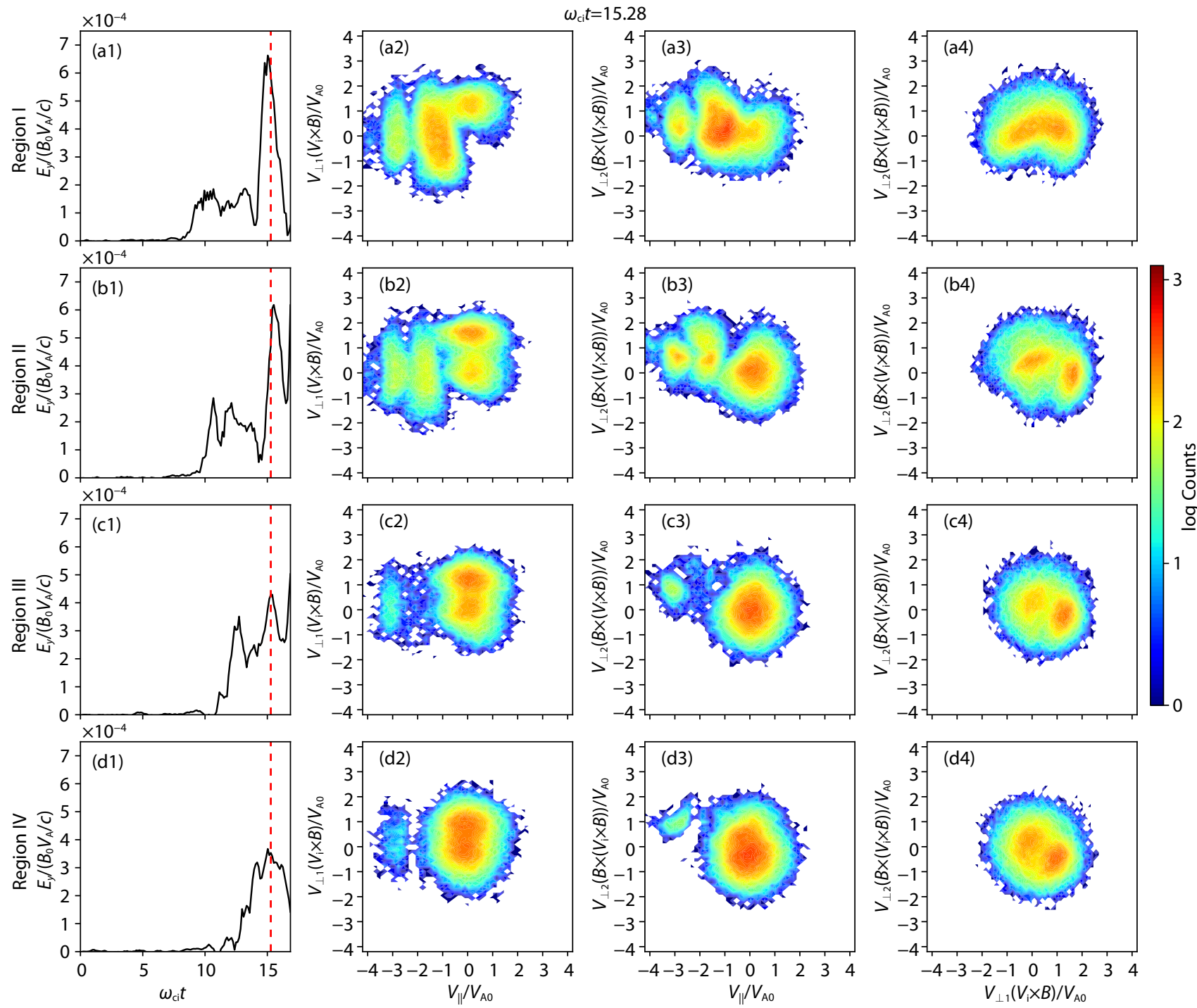

Figure 3. The spatial evolution of ion velocity distribution at $\omega_{\mathrm{ci}} t=15.28$. The velocity magnitude is normalized to $V_{\mathrm{AO}}$. The first column represents the intensity of the Hall electric field $E_{y}$ (normalized to $B_{0} V_{\mathrm{A} 0} / c$ ) in different regions. The red line indicates the current time when the distributions are taken. The rest columns are ion distributions taken from four locations indicated in Figure 1. Three arrays represent the reduced distributions on the $V_{\|}-V_{\perp 1}$ plane, the $V_{\|}-V_{\perp 2}$ plane and the $V_{\perp 1}-V_{\perp 2}$ plane respectively.

separatrix. For instance, at $\omega_{\mathrm{ci}} t=11$, Region II is actually outside the separatrix, thus there is a dip of the Hall electric field $E_{y}$ in Region II this time. Although the Hall electric field $E_{y}$ does not directly evolve from Region I to Region IV, we can roughly infer the cumulative effects that the reconnection exerts on the particles from the time variation of the Hall electric field $E_{y}$ in a certain region. Basically, the further away from the source region, the less the particles are influenced by the Hall electric field $E_{y}$. In other words, at $\omega_{\mathrm{ci}} t=15.28$, the particles in Region I have experienced long-time effects of the Hall electric field $E_{y}$ while the particles in Region IV have just started to be influenced by the Hall electric field. From the second column, in the $V_{\|}-V_{\perp 1}$ plane, we find that in Region IV, due to the little effect of the Hall electric field $E_{y}$ (Figure 3(d1)), the ion distribution splits into two parts: the main population is slightly deviated from the initial Maxwellian distribution and there is a visible population in the parallel direction (at
$V_{\|}=-2.8 V_{\mathrm{A} 0}$, Figure $\left.3(\mathrm{~d} 2)\right)$. In Region III, the main population is prone to split and the population in the parallel direction is being assembled (at $V_{\|}=-3.1 V_{\mathrm{A} 0}$, Figure 3(c2)). In Region II, a counterstreaming distribution is developing along the $V_{\perp 1}$ direction, and another two populations appear to be accelerated to two energy levels in the parallel direction. The ion distribution in Region I exhibits three discrete populations in the $V_{\|}$direction (Figure 3(a2)), the first one at $V_{\|}=0.0 V_{\mathrm{A} 0}$, the second at $V_{\|}=-1.5 V_{\mathrm{A} 0}$, and the third at $V_{\|}=-3.1 V_{\mathrm{A} 0}$. The above counter-streaming velocity distribution on the separatrices has been reported previously by observations (Wygant et al., 2005) and simulations (Aunai et al., 2011), and it is usually explained as the result of the bounce motion between separatrices. From the third column, which shows the ion velocity distribution in the $V_{\|}-V_{\perp 2}$ plane, we can see that the distinct signature of the ion distributions is mainly in the parallel direction, with overall enlargement and slight deviation from the 
central core in the $V_{\perp 2}$ direction, suggesting a heating process in the $V_{\perp 2}$ direction. The last column in Figure 3 indicates non-gyrotropic ion velocity distributions in the $V_{\perp 1}-V_{\perp 2}$ plane, with discrete populations in the $V_{\perp 1}$ direction and an enlarged distribution in the $V_{\perp 2}$ direction. Actually, the signatures in the $V_{\perp 1}-V_{\perp 2}$ space are implicitly expressed in the left columns.

Besides the two well-known counter-streaming populations in the $V_{\perp 1}$ direction, we find new populations in this simulation in the parallel direction, as shown in the Region II of Figure 3(b2). So next we investigate the ion velocity distribution at this location at different moments, as shown in Figure 4. As mentioned above, the velocity distribution is mainly affected by the Hall electric field $E_{y}$ on the separatrix. At $\omega_{\mathrm{ci}} t=10.91$ (Figure 4(e2)), when the Hall fields enhance just in Region II, the ion distribution is slightly deviated from its initial Maxwellian distribution. At $\omega_{\mathrm{ci}} t=13.1$ (Figure 4(f2)), some particles start to form a population in the parallel direction. At $\omega_{\mathrm{ci}} t=14.31$ (Figure 4(g2)), two populations in the parallel direction are about to form and the original core popula-
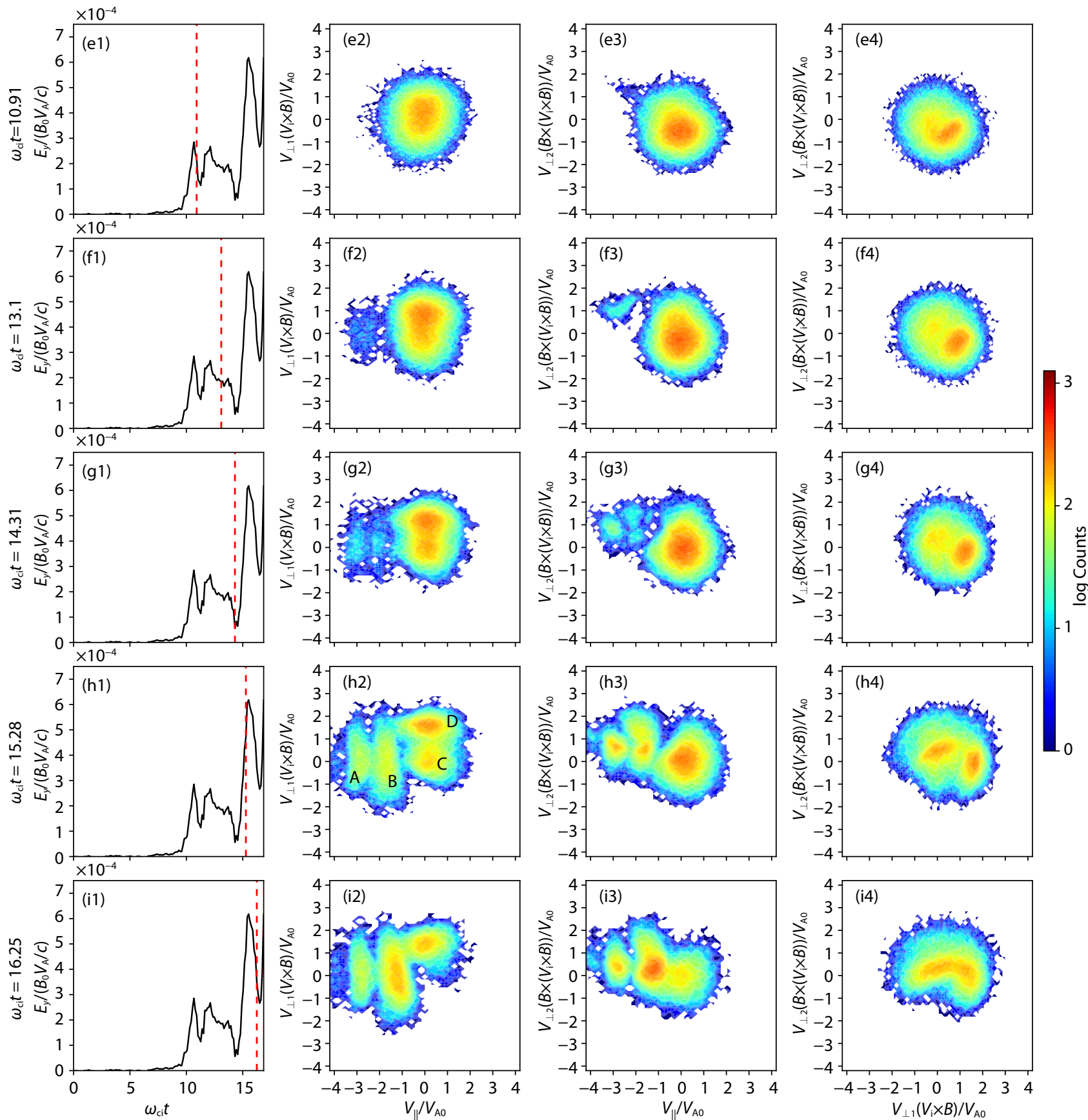

Figure 4. The ion velocity distribution taken from Region II at different moments. The velocity magnitude is normalized to $V_{\mathrm{A} 0}$. The first column represents the intensity of the Hall electric field $E_{y}$ (normalized to $B_{0} V_{\mathrm{A} 0} / c$ ). The red line indicates the time when the distributions are taken for each row. The distributions at each row represent the reduced distributions on the $V_{\|}-V_{\perp 1}$ plane, the $V_{\|}-V_{\perp 2}$ plane and the $V_{\perp 1}-V_{\perp 2}$ plane respectively. 
tion is about to split. At $\omega_{\text {ci }} t=15.28$ (Figure 4(h2)), two counterstreaming populations are developed in the $V_{\perp 1}$ direction, with one population centered at $V_{\perp 1}=1.7 V_{\mathrm{A} 0}$ and another at $V_{\perp 1}=-0.4 V_{\mathrm{A} 0}$. Meanwhile, two populations develop in the negative $V_{\|}$direction: a higher-energy one centered at $V_{\|}=-3.1 V_{\mathrm{A} 0}$ and a lower-energy one at $V_{\|}=-1.8 V_{\mathrm{A} 0}$. Such a distribution evolves into three discrete components at $\omega_{\text {ci }} t=16.25$ (Figure 4(i2)). The ion velocity in the $V_{\|}-V_{\perp 2}$ plane shows that the velocity distribution exhibits three discrete populations in the parallel direction. It should be noted that, given the short time interval, the above analysis of the velocity distribution can only roughly reflect the local variation of the particle distribution, and the involved particles at this particular location are not completely the same at these times.

\subsection{The Origins of the lon Distribution}

To illustrate how the above distributions are formed and where the particles originate from, we trace the particles in the simulation. We focus on the distribution observed in Region II at $\omega_{\text {ci }} t=15.28$ and trace backward to the initial state. The four populations in Figure $4(\mathrm{~h} 2)$ are noted as $A, B, C$, and D. Populations A and $B$ are energized at the negative $V_{\|}$direction, with $A$ at higher energies and $B$ at lower energies. Populations $C$ and $D$ are discrete in the $V_{\perp 1}$ direction, with $C$ at low energies and $D$ at high energies. Within each population, we randomly select 1000 ions to trace backward in time; we obtain their statistical trajectories. These particles are different from the common 'test particles' that do not participate in the self-consistent circulation. Instead, these particles are those that are involved in the dynamics of the plasma and providing feedback effects on the electromagnetic fields. We average their trajectories over a gyroperiod (the initial gyroperiod), superposed on the Hall electric field $E_{y}$ at $\omega_{\mathrm{ci}} t=15.28$, as shown in Figure 5.

Figure 5a shows the trajectories of particles in Population A. The red circle denotes the final destination of the tracing (i.e., Region II). Among the 1000 trajectories, we identify two typical kinds, representing two distinct sources. They are marked as Beams $A_{1}$ and $A_{2}$ respectively, with dark black lines. Although both beams are located initially at $Y<0$, one (Beam $A_{1}$ ) originates from the lower left quarter of the separatrices, i.e., $X<0$; the other (Beam $A_{2}$ ) comes from the lower right quarter, i.e., $X>0$. Particles represented by Beam $A_{1}$ possess positive $V_{x}$ (i.e., negative $V_{\|}$initially) so that they can arrive at Region II. They experience the reconnection through the inflow region and then enter the separatrix region, arriving at Region II. In contrast, particles represented by Beam $A_{2}$ initially possess a negative $V_{x}$ (i.e., positive $V_{\|}$). However, they, too, enter the inflow region as the reconnection evolves, and go through the reconnection process, finally arriving at Region II. As demonstrated in Part $C$, particles in Population A are constantly accelerated by the reconnection field $E_{z}$ during this kind of trajectory; therefore they gain the most energy of those in the distribution.

Population B also appears in the negative parallel direction in the distribution, meaning that they are accelerated in this direction but at lower energies compared to Population A. There are two evident beams in Population B, as shown in Figure 5c. They are initially located at the opposite sides of Region II. However, Beam $B_{1}$ originates from the upper left quarter of the separatrices, i.e., $X$ $<0$. These particles initially possess positive $V_{x}$ (positive $V_{\|}$due to the positive background magnetic field in the upper half of the simulation box). After they travel across the neutral sheet and arrive at the separatrix, the background magnetic field turns to the opposite direction. Thus they appear with negative $V_{\|}$in the specific distribution. On the other hand, particles within Beam $B_{2}$ initially possess a negative $V_{x}$ (i.e., negative $V_{\|}$). They are reflected from the separatrices on the opposite side, to the separatrices of interest. Aunai et al. (2011) suggested that the bounce motion between two separatrices is caused by the transfer between kinetic energy and electric potential energy, and is dominated by the electric force. In this case, they can be regarded as possessing a positive $V_{x}$ and passing across the neutral sheet, becoming part of Population $\mathrm{B}$ in the negative $V_{\|}$direction.

The trajectories of Populations $C$ and D are similar, as shown in Figures $4 \mathrm{e}$ and $4 \mathrm{~g}$. Both populations follow typical trajectories. One beam is located initially in the region of $X<0$ in the inflow region, labeled as Beam $C_{1}$ or $D_{1}$; the other beam initially locates at the region of $X>0$ as Beam $C_{2}$ or $D_{2}$. Beam $C_{1}$ particles arrive at the separatrix earlier than Beam $D_{1}$ particles. After they cross the separatrix, they are bounced back to the separatrix and then arrive at Region II. Beam $D_{1}$ particles, however, arrive at Region II directly from the undisturbed inflow region (Figure $5 \mathrm{~h}$ ) at $\omega_{\mathrm{ci}} t=15.28$. On the other hand, Beams $C_{2}$ and $D_{2}$ initially move in the $-X$ direction toward Region II. Overall, Beam $C_{2}$ is initially closer to the neutral sheet than is Beam $D_{2}$. In fact, most particles in Beam $D_{2}$ resemble those of Beam $D_{1}$, which are directly from the undisturbed inflow region, as will be shown in Figure $6 \mathrm{~d}$.

To understand better the differences among these four populations, Figure 6 shows the statistical $Y$ location of their trajectory in time. It can be seen that Beams $A_{2}, B_{1}, C_{1}$, and $D_{1}$ dominate the Populations $A, B, C$, and $D$, respectively. The red dashed horizontal line indicates the location of Region II, while the vertical red dashed line indicates the moment at $\omega_{\mathrm{ci}} t=15.28$. It can be seen that all the particles in Population A initially locate at the region of $Y<0$, but they all cross the neutral sheet $(Y=0)$ around $\omega_{\mathrm{ci}} t=11.8$. While all particles in Population $B$ initially locate at the region of $Y>0$, indicating that Population $B$ is composed of particles originating from the opposite side of the separatrix, they also cross the neutral sheet $Y=0$ around $\omega_{\mathrm{ci}} t=14$. Obviously, particles in Population $A$ are involved in the reconnection process earlier than those of Population $B$ while moving from the $X$ line towards the outflow region. For Population $C$ and Population $D$, all particles are initially at the same side of the separatrix, i.e., at the region of $Y<0$. It can be seen from Figure $6 \mathrm{c}$ and $6 \mathrm{~d}$ that Population $C$ crosses the separatrix as early as $\omega_{\mathrm{ci}} t=13-14$, while most particles in Population $\mathrm{D}$ arrive for the first time at $\omega_{\mathrm{ci}} t=15.28$. Figure 6 also shows the subsequent stage for these particles. After $\omega_{\mathrm{ci}} t=15.28$, almost all the particles migrate to positive $Y$ locations, indicating that particles are reflected by the separatrix into the exhaust region.

\subsection{Energy Conversion on the Separatrices}

We further examine how the energy of these ions changes during the development of this distribution. Here we focus on the en- 

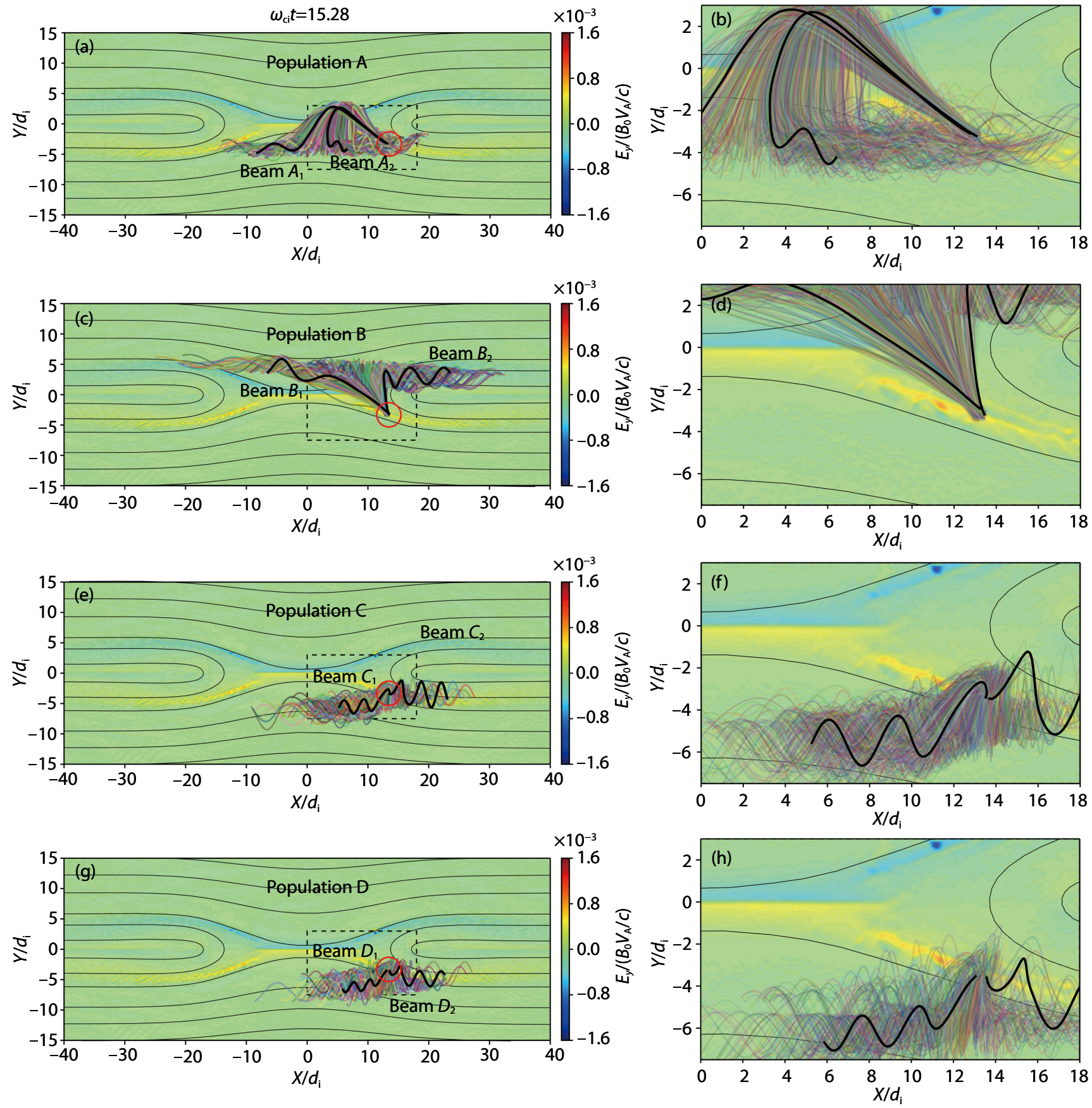

Figure 5. Trajectories of representative particles within the ion distribution are shown with the Hall electric field $E_{y}$ (normalized to $B_{0} V_{\mathrm{A} 0} / c$ ) at $\omega_{\mathrm{ci}} t=15.28$ superposed on it. The light black lines indicate the magnetic field lines. Four populations indicated in Figure 4(h2) are shown respectively. The dark lines show the typical motion of ions. The red circle denotes the Region II. The zoom-in regions denoted with dashed rectangles are shown in the right columns.

ergy conversion in the inertial frame. According to the Poynting theorem, the term $J \cdot E$ represents the energy conversion between electromagnetic energy and plasma kinetic energy. When $J \cdot E>0$, the electric fields transfer their energy to the particles. Conversely, when $J \cdot E<0$, energy is transferred from plasma to the fields. As shown in Figure 7 , three components of $J_{i} \cdot E$ contributing to ion energization dominate in different regions. Along the separatrix, the main term in energy conversion is $J_{i y} E_{y}$, meaning that the Hall electric field $E_{y}$ contributes to the energization of particles. The contribution of $J_{\mathrm{ix}} E_{x}$ along the separatrix is mainly confined near the $X$ line, while the effect of $J_{i z} E_{z}$ is slightly negative. In the outflow region enclosed by the separatrices, $J_{\mathrm{iz}} E_{z}$ dominates the ion energization process, which is contributed by the out-of-plane reconnection electric field $E_{z}$.

The above different populations, originating from different locations and going through different paths, are naturally subject to 

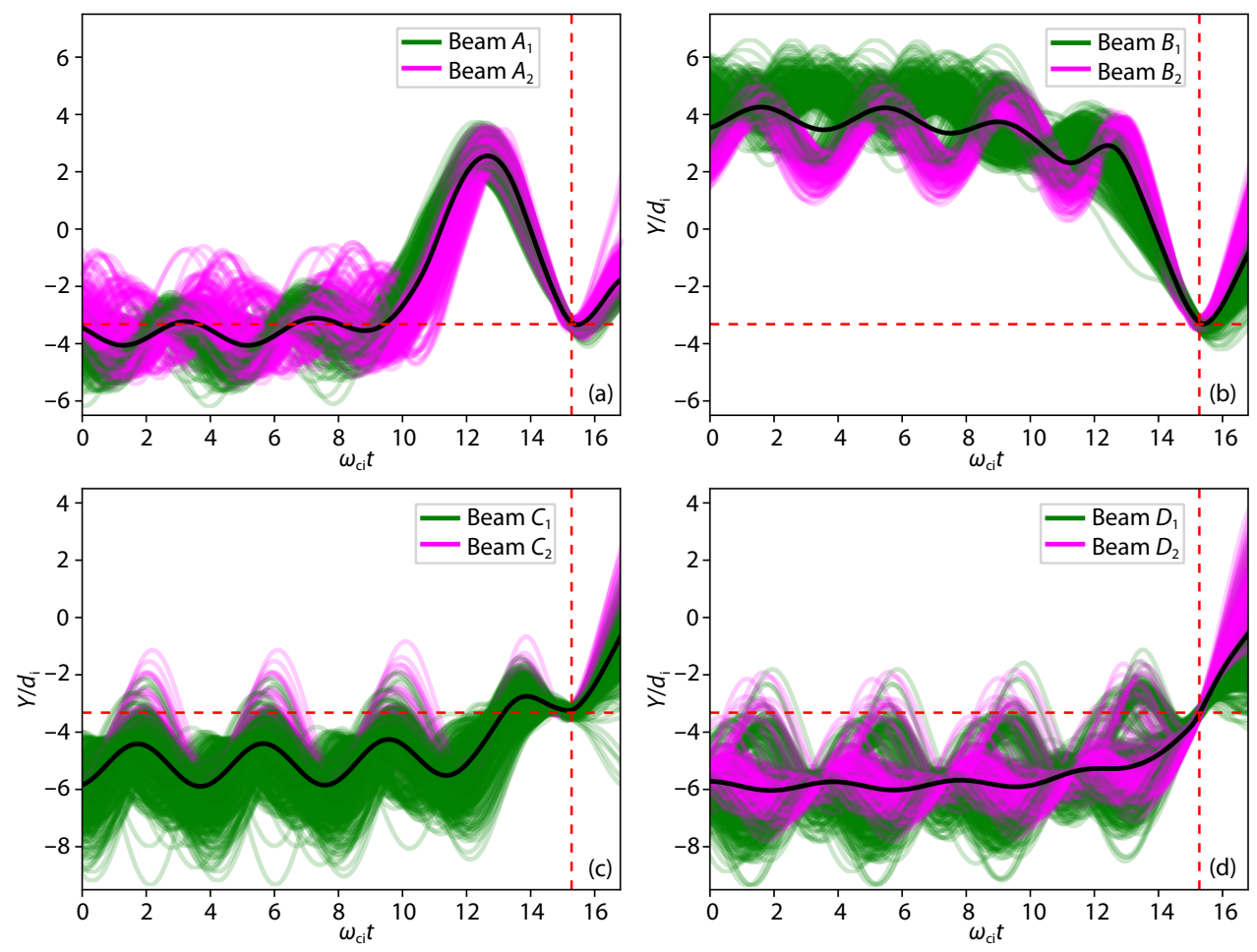

Figure 6. Statistical location of particles in Populations A, B, C, and D. Each particle is represented by a colorful line. The dark line represents the average property for all the particles. The vertical red dashed line indicates the moment $\omega_{\mathrm{ci}} t=15.28$. The horizontal red dashed line indicates the location of Region II.

these electric fields. Populations A and B are originally from the same side or the opposite side of the separatrix. Both of them cross the neutral sheet to arrive at the separatrix and gain energy from the in-plane electric field $E_{x}$ and the Hall electric field $E_{y}$ on the separatrix. During this process, the Hall electric field $E_{y}$ on the separatrix also serves as the potential well that contributes the bounce motion by transferring kinetic energy between $\left|V_{x}\right|$ and $\left|V_{y}\right|$ (Aunai et al., 2011). However, particles in Population A cross the neutral sheet earlier and are constantly accelerated by the reconnection electric field $E_{z}$, forming the population with the highest energy in the negative parallel direction. Particles in Population $B$ arrive at the separatrix from the opposite side of the separatrices instead of the reconnection region. They do not experience $E_{z}$, resulting in less acceleration. This leads to the formation of the two populations with negative $V_{\|}$in the specific distribution. The two counter-streaming populations along the perpendicular direction are composed mainly of particles originating from the undisturbed inflow region on the same side of the separatrix. Particles in Population $C$ travel across the separatrix in advance and then turn around to the separatrix again towards the location of interest, while those in Population D just arrive at the separatrix in time. Although all of them are accelerated by the Hall electric field $E_{y}$ on the separatrix, Population $C$ enters the outflow region through the separatrix and is decelerated by the induced electric field $E_{z}$. This is the reason for the formation of counterstreaming distribution with low-energy and high-energy populations.

Figure 8 shows the temporal variation of the energy of Popula- tions $A, B, C$, and $D$ as well as the work done by different electric fields $W_{E_{x}}, W_{E_{y}}$ and $W_{E_{z}}$ through integrating $E_{x}, E_{y}$ and $E_{z}$ along the particle trajectory. Population A originating from the same side of the separatrix and crossing the neutral sheet around $\omega_{\mathrm{ci}} t=11.8$, is accelerated by the in-plane electric field $E_{x}$ (Figure 8(a2)), and the Hall electric field $E_{y}$ (Figure 8(a3)) before arriving at Region II. This corresponds to the energy enhancement in Figure 8(a1) between $\omega_{\mathrm{ci}} t=10-12$. Moreover, it is obvious that Population A is constantly energized by the reconnection electric field $E_{z}$ since it crosses the neutral sheet (Figure 8(a4)).

Similarly, Population B, originating from the opposite side of the separatrix and crossing the opposite separatrix, is accelerated by the in-plane electric field $E_{x}$ and the Hall electric field $E_{y}$ (Figure 8(b1)). Unlike Population A, Population B is barely affected by the reconnection electric field $E_{z}$ (Figure $8(\mathrm{~b} 4)$ ). This is the reason that the total energy of Population B is lower than that of Population A.

Population C crosses the separatrix before $\omega_{\mathrm{ci}} t=15.28$, and is accelerated by the Hall electric field $E_{y}$ along the separatrix. Thus the energy of Population $C$ increases before $\omega_{\text {ci }} t=15.28$. Note that after Population $C$ crosses the separatrix, but has not yet arrived near the neutral sheet, it is decelerated by the induced electric field $E_{z}$ (Figure 8(c4)). This is because the $J_{\mathrm{iz}} E_{z}$ is negative slightly above the separatrix.

Population $D$, originating from the unperturbed plasma, does not arrive at the separatrix until $\omega_{\mathrm{ci}} t=15.28$ (Figure 6(d)). As a result, the energy for Population $D$ does not change much until $\omega_{\mathrm{ci}} t=15.28$ when it starts to increase sharply under the influence 

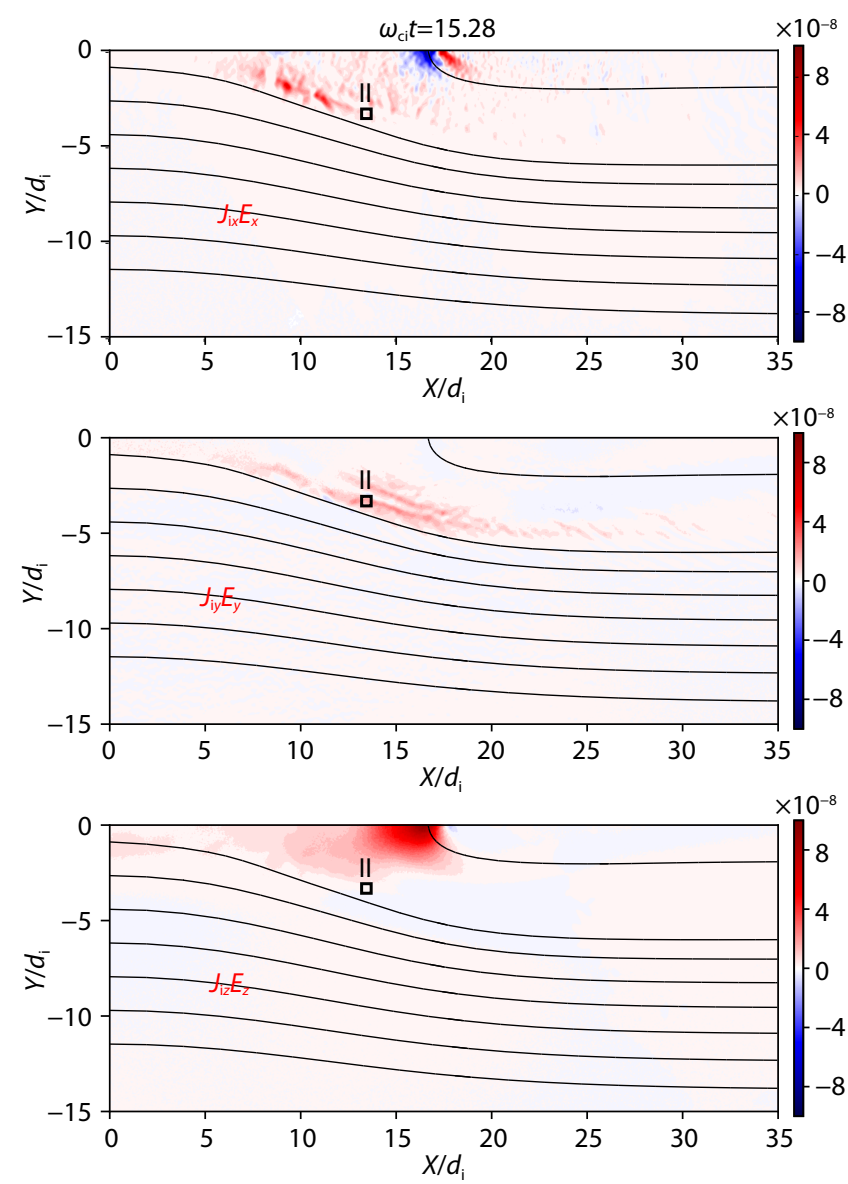

Figure 7. Energy conversion terms along and above the separatrix. Electric field is normalized to $B_{0} V_{\mathrm{A} 0} / c$, and ion current is normalized to $e n_{0} c$. Three components of $J \cdot E$ are shown along the separatrix and above the separatrix respectively. Region II for detailed analysis of ion behaviors is marked with a black rectangle.

of Hall electric field $E_{y}$ on the separatrix.

To summarize, for ions that arrive at the separatrix, the roles of the three electric field components are quite different. The in-plane electric field $E_{x}$ on the separatrices always accelerate ions when they pass through the separatrix. The Hall electric field $E_{y}$ serves as an accelerator for ions that come from the unperturbed plasma. But for ions that bounce within the separatrices, it contributes to the bounce motion by acting as the potential well. The induced electric field $E_{z}$ is another efficient accelerator for ions crossing the neutral sheet in the reconnection process. However, it decelerates ions that cross the separatrix but have not arrived in the region of the neutral sheet.

\section{Conclusion and Discussion}

Separatrices are important sites in the magnetic reconnection for the occurrence of multiple waves and the potential to accelerate particles. Recent studies have revealed that, in magnetic reconnection, ions gain more energy than do electrons. In this study, by means of 2-D particle-in-cell simulations, we have studied the ion distributions on the separatrices, the origins of these populations, and their energetics. The main findings are summarized as follows.

(1) The separatrices are developed from the X point, dividing the unreconnected from the reconnected plasma. We exhibit the ion distributions at different regions on the separatrices and at different reconnection stages. The ion velocity distributions at different regions and moments reveal the existence of a specific velocity distribution: two counter-streaming core populations in the perpendicular direction and two discrete populations in the negative parallel direction. The counter-streaming cores are not symmetric in the perpendicular direction; they exhibit different energy levels. The two extra populations are also separately energized into two different energy levels in the negative parallel direction.

(2) Through investigation of the trajectories of the ions, the origins of such a distribution are revealed. The higher-energy population at the negative parallel direction is found to be composed of ions originally located on the same side of the separatrix of interest. All particles in this population are involved in the reconnection process and travel across the neutral sheet. The lower-energy population at the negative parallel direction is originally located on the opposite side of the separatrix. These particles move across the opposite separatrix and arrive at the separatrix of interest. The counter-streaming cores are discretized into two parts along the perpendicular direction. Although both are located initially mostly at the same side of the separatrix, the particles with higher energy enter the separatrix earlier than the population with lower energy that comes directly from the undisturbed plasma.

(3) Along the separatrix, the energy gain of the particles is attributable primarily to the Hall electric field. In the outflow region enclosed by the separatrices, however, it is the reconnection electric field that dominates the energization process. Along the paths to the separatrix, the two populations that gain energy in the negative parallel direction are energized by both the in-plane electric field and the Hall electric field, but the population with higher energy gains additional energy from the reconnection electric field. During the development of the counter-streaming distribution, particles travel across the separatrix and are influenced by both the Hall electric field on the separatrix and the reconnection electric field between the two separatrices. Nevertheless, the main contributor to the ion energy gain is the Hall electric field.

It should be noted that this simulation is idealized in some aspects. For instance, the artificial mass ratio $m_{\mathrm{i}} / m_{\mathrm{e}}=100$ is employed. Although this simplification has been used widely in previous studies, Gurram et al. (2020) have suggested that the Hall fields are over-damped due to electron Landau damping in the case with low mass ratio $\left(m_{\mathrm{i}} / m_{\mathrm{e}}=25\right)$ compared to the case with a higher mass ratio $\left(m_{\mathrm{i}} / m_{\mathrm{e}}=400\right)$. The Hall fields in the situation where the value of the mass ratio is natural (i.e., $m_{\mathrm{i}} / m_{\mathrm{e}}=1836$ ) are believed to propagate to a larger domain. In that case, ions from the unperturbed plasma are capable of attaining higher energy from the Hall electric field. Meanwhile, we caution that this study has investigated a 2-D symmetric setting without guide field, whereas magnetic reconnection occurring in space plasmas usually contains a non-negligible guide field. The guide field will res- 

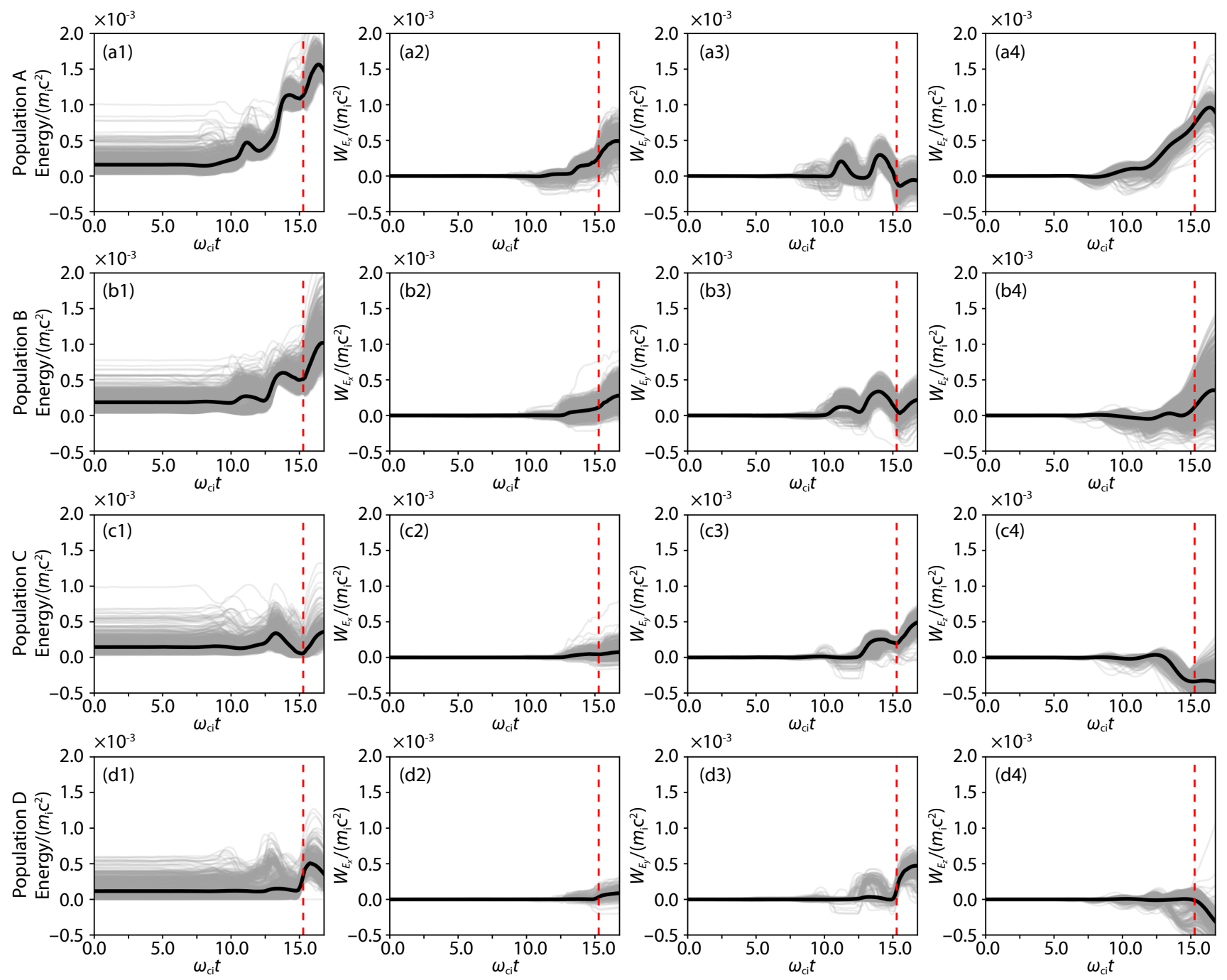

Figure 8. Statistical energy of particles in Population $A, B, C$ and $D$ along the particle path. The four columns respectively show total energy (normalized to $m_{0} c^{2}$ ), work (normalized to $m_{0} c^{2}$ ) done by $E_{x}, E_{y}$ and $E_{z}$. Each light black line represents a particle. The dark line represents the average property for all the particles. The vertical red dashed line indicates the moment $\omega_{\mathrm{ci}} t=15.28$.

ult in distortion of symmetric Hall fields. Fu $S$ et al. (2018) suggested that, when the guide field is larger than $0.3 B_{0}$, a new electric field with opposite polarity will appear on the separatrix. Taking such an effect into account can be expected to lead to a more complex picture of the ion trajectory around the separatrix. We note further that this study does not consider the various waves excited on the separatrices (Fujimoto, 2014), nor the waves and the instabilities that are excited in the reconnection due to the 3-D geometry. These issues and limitations will be tackled gradually in our future investigations.

\section{Acknowledgments}

This work was supported by the NSFC grants 41821003 and 41974192, by the B-type Strategic Priority Program of the Chinese Academy of Sciences (Grant No. XDB41000000), and by the pre-research projects on Civil Aerospace Technologies No. D020103 funded by China's National Space Administration (CNSA). We also thank Dr. Shan Wang for helpful discussions. Simulations were performed on TianHe-2 at National Supercomputer Center in Guangzhou, China. The simulation code iPIC3D is available at https://bitbucket.org/bopkth/ipic3d-klm.

\section{References}

Aunai, N., Belmont, G., and Smets, R. (2011). Proton acceleration in antiparallel collisionless magnetic reconnection: kinetic mechanisms behind the fluid dynamics. J. Geophys. Res.: Space Phys., 116(A9), A0932. https://doi.org/10.1029/2011JA016688

Burch, J. L., Torbert, R. B., Phan, T. D., Chen, L. J., Moore, T. E., Ergun, R. E., Eastwood, J. P., Gershman, D. J., Cassak, P. A., ... Chandler, M. (2016). Electron-scale measurements of magnetic reconnection in space. Science, 352(6290), aaf2939. https://doi.org/10.1126/science.aaf2939

Chen, L. J., Hesse, M., Wang, S., Bessho, N., and Daughton, W. (2016). Electron energization and structure of the diffusion region during asymmetric reconnection. Geophys. Res. Lett., 43(6), 2405-2412. https://doi.org/10.1002/2016GL068243

Dai, L. (2009). Collisionless magnetic reconnection via Alfvén eigenmodes. Phys. Rev. Lett., 102(24), 245003. https://doi.org/10.1103/PhysRevLett.102.245003 Dai, L., Wang, C., Zhang, Y. C., Lavraud, B., Burch, J., Pollock, C., and Torbert, R. B. 
(2017). Kinetic Alfvén wave explanation of the Hall fields in magnetic reconnection. Geophys. Res. Lett., 44(2), 634-640.

https://doi.org/10.1002/2016GL071044

Dai, L. (2018). Structures of hall fields in asymmetric magnetic reconnection. J. Geophys. Res.: Space Phys., 123(9), 7332-7341.

https://doi.org/10.1029/2018JA025251

Daughton, W., Roytershteyn, V., Karimabadi, H., Yin, L., Albright, B. J., Bergen, B., and Bowers, K. J. (2011). Role of electron physics in the development of turbulent magnetic reconnection in collisionless plasmas. Nat. Phys., 7(7), 539-542. https://doi.org/10.1038/nphys1965

Drake, J. F., Shay, M. A., Thongthai, W., and Swisdak, M. (2005). Production of energetic electrons during magnetic reconnection. Phys. Rev. Lett., 94(9), 095001. https://doi.org/10.1103/PhysRevLett.94.095001

Drake, J. F., Swisdak, M., Phan, T. D., Cassak, P. A., Shay, M. A., Lepri, S. T., Lin, R. P., Quataert, E., and Zurbuchen, T. H. (2009). Ion heating resulting from pickup in magnetic reconnection exhausts. J. Geophys. Res.: Space Phys. 114(A5), A05111. https://doi.org/10.1029/2008JA013701

Dungey, J. W. (1961). Interplanetary magnetic field and the auroral zones. Phys. Rev. Lett., 6(2), 47. https://doi.org/10.1103/PhysRevLett.6.47

Fu, H. S., Khotyaintsev, Y. V., Vaivads, A., André, M., Sergeev, V. A., Huang, S. Y., Kronberg, E. A., and Daly, P. W. (2012). Pitch angle distribution of suprathermal electrons behind dipolarization fronts: a statistical overview. J. Geophys. Res.: Space Phys., 117(A12), A12221.

https://doi.org/10.1029/2012JA018141

Fu, S., Huang, S. Y., Zhou, M., Ni, B. B., and Deng, X. H. (2018). Tripolar electric field Structure in guide field magnetic reconnection. Ann. Geophys., 36(2), 373-379. https://doi.org/10.5194/angeo-36-373-2018

Fu, X. R., Lu, Q. M., and Wang, S. (2006). The process of electron acceleration during collisionless magnetic reconnection. Phys. Plasmas, 13(1), 012309. https://doi.org/10.1063/1.2164808

Fujimoto, K. (2014). Wave activities in separatrix regions of magnetic reconnection. Geophys. Res. Lett., 41(8), 2721-2728. https://doi.org/10.1002/2014GL059893

Goldman, M. V., Newman, D. L., Lapenta, G., Andersson, L., Gosling, J. T., Eriksson, S., Markidis, S., Eastwood, J. P., and Ergun, R. (2014). Čerenkov emission of quasiparallel whistlers by fast electron phase-space holes during magnetic reconnection. Phys. Rev. Lett., 112(14), 145002. https://doi.org/10.1103/PhysRevLett.112.145002

Gurram, H., Egedal, J., and Daughton, W. (2020). Mode converting Alfvén waves from magnetic reconnection enhancing the energy source for the aurora borealis. arXiv preprint arXiv: 2004.11755.

Hesse, M., Aunai, N., Sibeck, D., and Birn, J. (2014). On the electron diffusion region in planar, asymmetric, systems. Geophys. Res. Lett., 41(24), 8673-8680. https://doi.org/10.1002/2014GL061586

Hoshino, M., Mukai, T., Terasawa, T., and Shinohara, I. (2001). Suprathermal electron acceleration in magnetic reconnection. J. Geophys. Res.: Space Phys., 106(A11), 25979-25997. https://doi.org/10.1029/2001JA900052

Huang, C., Wu, M. Y., Lu, Q. M., Wang, R. S., and Wang, S. (2015). Electron acceleration in the dipolarization front driven by magnetic reconnection. J. Geophys. Res.: Space Phys., 120(3), 1759-1765. https://doi.org/10.1002/2014JA020918

Huang, H. T., Yu, Y. Q., Dai, L., and Wang, T. Y. (2018). Kinetic Alfvén waves excited in two - dimensional magnetic reconnection. J. Geophys. Res.: Space Phys., 123(8), 6655-6669. https://doi.org/10.1029/2017JA025071

Huang, S. Y., Vaivads, A., Khotyaintsev, Y. V., Zhou, M., Fu, H. S., Retinò, A., Deng, X. H., André, M., Cully, M., ... Pang, Y. (2012). Electron acceleration in the reconnection diffusion region: cluster observations. Geophys. Res. Lett., 39(11), L11103. https://doi.org/10.1029/2012GL051946

Huang, S. Y., Fu, H. S., Yuan, Z. G., Vaivads, A., Khotyaintsev, Y. V., Retino, A., Zhou, M., Graham, D. B., Fujimoto, K., ... Zhou, X. (2016a). Two types of whistler waves in the hall reconnection region. J. Geophys. Res.: Space Phys., 121(7), 6639-6646. https://doi.org/10.1002/2016JA022650

Huang, S. Y., Retino, A., Phan, T. D., Daughton, W., Vaivads, A., Karimabadi, H., Zhou, W., Sahraoui, F., Li, G. L., ... Wang, D. D. (2016b). In situ observations of flux rope at the separatrix region of magnetic reconnection. J. Geophys.
Res.: Space Phys., 121(1), 205-213. https://doi.org/10.1002/2015JA021468

Huang, S. Y., Yuan, Z. G., Sahraoui, F., Fu, H. S., Pang, Y., Zhou, M., Fujimoto, K., Deng, X. H., Retinò, A., ... Li, H. M. (2017). Occurrence rate of whistler waves in the magnetotail reconnection region. J. Geophys. Res.: Space Phys., 122(7), 7188-7196. https://doi.org/10.1002/2016JA023670

Karimabadi, H., Daughton, W., and Scudder, J. (2007). Multi - scale structure of the electron diffusion region. Geophys. Res. Lett., 34(13), L13104. https://doi.org/10.1029/2007GL030306

Lapenta, G., Berchem, J., Zhou, M., Walker, R. J., El - Alaoui, M., Goldstein, M. L., Paterson, W. R., Giles, B. L., Pollock, C. J., ... Burch, J. L. (2017). On the origin of the crescent - shaped distributions observed by MMS at the magnetopause. J. Geophys. Res.: Space Phys., 122(2), 2024-2039. https://doi.org/10.1002/2016JA023290

Li, X. C., Guo, F., Li, H., and Li, G. (2015). Nonthermally dominated electron acceleration during magnetic reconnection in a low-beta plasma. Astrophys. J. Lett., 811 (2), L24. https://doi.org/10.1088/2041-8205/811/2/L24

Liang, J., Lin, Y., Johnson, J. R., Wang, X. Y., and Wang, Z. X. (2016). Kinetic Alfvén waves in three-dimensional magnetic reconnection. J. Geophys. Res.: Space Phys., 121(7), 6526-6548. https://doi.org/10.1002/2016JA022505

Markidis, S., Lapenta, G., and Rizwan-uddin. (2010). Multi-scale simulations of plasma with iPIC3D. Math. Comput. Simul., 80(7), 1509-1519. https://doi.org/10.1016/j.matcom.2009.08.038

Shay, M. A., Phan, T. D., Haggerty, C. C., Fujimoto, M., Drake, J. F., Malakit, K., Cassak, P. A., and Swisdak, M. (2016). Kinetic signatures of the region surrounding the $\mathrm{X}$ line in asymmetric (magnetopause) reconnection. Geophys. Res. Lett., 43(9), 4145-4154. https://doi.org/10.1002/2016GL069034

Wang, H. Y., Lu, Q. M., Huang, C., and Wang, S. (2016). The mechanisms of electron acceleration during multiple $X$ line magnetic reconnection with a guide field. Astrophys. J., 821(2), 84. https://doi.org/10.3847/0004637X/821/2/84

Wang, H. Y., Lu, Q. M., Huang, C., and Wang, S. (2017). Electron acceleration in a secondary magnetic island formed during magnetic reconnection with a guide field. Phys. Plasmas, 24(5), 052113. https://doi.org/10.1063/1.4982813

Wang, R. S., Du, A. M., Nakamura, R., Lu, Q. M., Khotyaintsev, Y. V., Volwerk, M., Zhang, T. L., Kronberg, E. A., Daly, P. W., and Fazakerley, A. N. (2013). Observation of multiple sub-cavities adjacent to single separatrix. Geophys. Res. Lett., 40(11), 2511-2517. https://doi.org/10.1002/grl.50537

Wang, S., Chen, L. J., Hesse, M., Bessho, N., Gershman, D. J., Dorelli, J., Giles, B., Torbert, R. B., Pollock, C. J., ... Saito, Y. (2016). Two-scale ion meandering caused by the polarization electric field during asymmetric reconnection. Geophys. Res. Lett., 43(15), 7831-7839. https://doi.org/10.1002/2016GL069842

Wang, S., Chen, L. J., Bessho, N., Hesse, M., Yoo, J., Yamada, M., Liu, Y. H., Gershman, D. J., Giles, B., and Moore, T. E. (2018). Energy conversion and partition in the asymmetric reconnection diffusion region. J. Geophys. Res.: Space Phys., 123(10), 8185-8205. https://doi.org/10.1029/2018JA025519

Wygant, J. R., Cattell, C. A., Lysak, R., Song, Y., Dombeck, J., McFadden, J., Mozer, F. S., Carlson, C. W., Parks, G., ... Mouikis, C. (2005). Cluster observations of an intense normal component of the electric field at a thin reconnecting current sheet in the tail and its role in the shock-like acceleration of the ion fluid into the separatrix region. J. Geophys. Res.: Space Phys., 110(A9), A09206. https://doi.org/10.1029/2004JA010708

Yamada, M., Kulsrud, R., and Ji, H. T. (2010). Magnetic reconnection. Rev. Mod. Phys., 82(1), 603. https://doi.org/10.1103/RevModPhys.82.603

Yamada, M., Yoo, J., Jara-Almonte, J., Ji, H. T., Kulsrud, R. M., and Myers, C. E. (2014). Conversion of magnetic energy in the magnetic reconnection layer of a laboratory plasma. Nat. Commun., 5, 4774. https://doi.org/10.1038/ncomms5774

Zenitani, S., Hesse, M., Klimas, A., and Kuznetsova, M. (2011). New measure of the dissipation region in collisionless magnetic reconnection. Phys. Rev. Lett., 106(19), 195003. https://doi.org/10.1103/PhysRevLett.106.195003

Zhou, M., Ashour-Abdalla, M., Deng, X. H., Schriver, D., El-Alaoui, M., and Pang, Y. (2009). THEMIS observation of multiple dipolarization fronts and associated wave characteristics in the near-Earth magnetotail. Geophys. Res. Lett., 36(20), L20107. https://doi.org/10.1029/2009GL040663 
Zhou, M., Deng, X. H., Pang, Y., Huang, S. Y., Yuan, Z. G., Li, H. M., Xu, X. J., Wang, Y. H., Yan, M., and Wang, D. D. (2012). Revealing the sub-structures of the magnetic reconnection separatrix via particle-in-cell simulation. Phys. Plasmas, 19(7), 072907. https://doi.org/10.1063/1.4739283

Zhou, X. Z., Angelopoulos, V., Runov, A., Sitnov, M. I., Zong, Q. G., and Pu, Z. Y. (2009). Ion distributions near the reconnection sites: comparison between simulations and THEMIS observations. J. Geophys. Res.: Space Phys., 114(A12), A12211. https://doi.org/10.1029/2009JA014614
Zhou, X. Z., Angelopoulos, V., Sergeev, V. A., and Runov, A. (2010). Accelerated ions ahead of earthward propagating dipolarization fronts. J. Geophys. Res.: Space Phys., 115(A5), A00I03. https://doi.org/10.1029/2010JA015481

Zhou, X. Z., Pan, D. X., Angelopoulos, V., Runov, A., Zong, Q. G., and Pu, Z. Y. (2016). Understanding the ion distributions near the boundaries of reconnection outflow region. J. Geophys. Res.: Space Phys., 121(10), 9400-9410. https://doi.org/10.1002/2016JA022993 